\section{Síndrome hemofagocítico asociado con hepatitis}

\author{
Eunice Sandoval-Ramírez ${ }^{1}$, Ignacio Camacho-Meza ${ }^{1}$, Nery Eduardo-Solís ${ }^{1}$, \\ Oswaldo Plascencia-Tabares ${ }^{2}$, Efraín Navarro-Olivos ${ }^{3}$, Francisco Ignacio \\ Ortiz-Aldana $^{3}$
}

\section{Resumen}

El síndrome hemofagocítico se distingue por la proliferación y activación de células presentadoras de antígeno en la médula ósea y otros órganos del sistema retículo endotelial, así como de linfocitos T CD8+ que ponen en peligro la vida de los pacientes. Las manifestaciones clínicas predominantes, como fiebre, citopenias, hepatitis, coagulopatía, síntomas neurológicos e insuficiencia orgánica múltiple están relacionadas con inflamación sistémica. Comunicamos el caso de un lactante que inició su padecimiento con ictericia, dolor abdominal, vómito, ataque al estado general, hepatomegalia, esplenomegalia y características bioquímicas sugerentes de inflamación hepatocelular y colestasis progresiva con mala evolución clínica; al cuadro se agregó fiebre persistente, crisis convulsivas, anemia, trombocitopenia, leucopenia, ferritina y triglicéridos elevados, que integraron síndrome hemofagocítico con desenlace fatal a pesar de recibir tratamiento inmunosupresor.

PALABRAS CLAVE: síndrome, hemofagocitosis, hepatitis, citocinas, macrófago.

Rev Alerg Méx 2016 Jan-Mar;63(1):91-94.

\section{Hemophagocytic syndrome associated to hepatitis.}

Eunice Sandoval-Ramírez ${ }^{1}$, Ignacio Camacho-Meza ${ }^{1}$, Nery Eduardo-Solís ${ }^{1}$, Oswaldo Plascencia-Tabares ${ }^{2}$, Efraín Navarro-Olivos ${ }^{3}$, Francisco Ignacio Ortiz-Aldana ${ }^{3}$

\begin{abstract}
Hemophagocytic syndrome is characterized by increased proliferation and activation of antigen presenting cells (histiocytes) in bone marrow and other organs of the reticuloendothelial system as well as CD8+ T cells that threatens life of patients. The predominant clinical manifestations such as fever, cytopenia, hepatitis, coagulopathy, neurological symptoms and multiple organ failure are related to systemic inflammation. We report the case of an infant who started with jaundice, abdominal pain, vomiting and malaise, at admission, hepatomegaly, splenomegaly and biochemically with features suggestive of hepatocellular inflammation and progressive cholestasis with poor outcome, it was added persistent fever, seizures, anemia, thrombocytopenia, leukopenia, elevated ferritin and hypertriglyceridemia integrating hemophagocytic syndrome with fatal outcome despite immunosuppressive therapy.
\end{abstract}

KEYWORDS: syndrome; hemophagocytosis; hepatitis; cytokine; macrophage

\footnotetext{
${ }^{1}$ Servicio de Alergia e Inmunología Clínica Pediátrica. 2 Servicio de Medicina del Enfermo Pediátrico en Estado Crítico.

Hospital de Especialidades Pediátrico de León, Guanajuato, México.

${ }^{3}$ Instituto de Salud Pública del Estado de Guanajuato, Guanajuato, México.
}

Recibido: 2 de septiembre 2015

Aceptado: 8 de diciembre 2015

Correspondencia

Dra. Eunice Sandoval Ramírez

drasandoval83@yahoo.com

Este artículo debe citarse como

Sandoval-Ramírez E, Camacho-Meza I, Eduardo-Solís $\mathrm{N}$, Plascencia-Tabares $\mathrm{O}$ y col. Síndrome hemofagocítico asociado con hepatitis. Rev Alerg Méx. 2016 ene-mar;63(1):91-94. 


\section{ANTECEDENTES}

El síndrome hemofagocítico, sea primario o secundario, se distingue por proliferación y activación de células presentadoras de antígeno y linfocitos T CD8+ en la médula ósea y otros órganos del sistema retículo endotelial que ponen en peligro la vida de los pacientes. ${ }^{1}$ Las manifestaciones clínicas predominantes, como fiebre, citopenias, hepatitis, coagulopatía y síntomas neurológicos, están relacionadas con inflamación sistémica. ${ }^{2}$

Entre las causas primarias del síndrome se incluyen la linfohisticitosis hemofagocítica familiar y el síndrome linfoproliferativo ligado al cromosoma $X$, mientras que las secundarias incluyen las infecciones, fármacos, procesos malignos y autoinmunitarios (Cuadro 1). ${ }^{3}$ El diagnóstico se relaciona con sospecha de sepsis, lo que retrasa el tratamiento. En 2004 la Sociedad del

Cuadro 1. Procesos infecciosos asociados con síndrome hemofagocítico

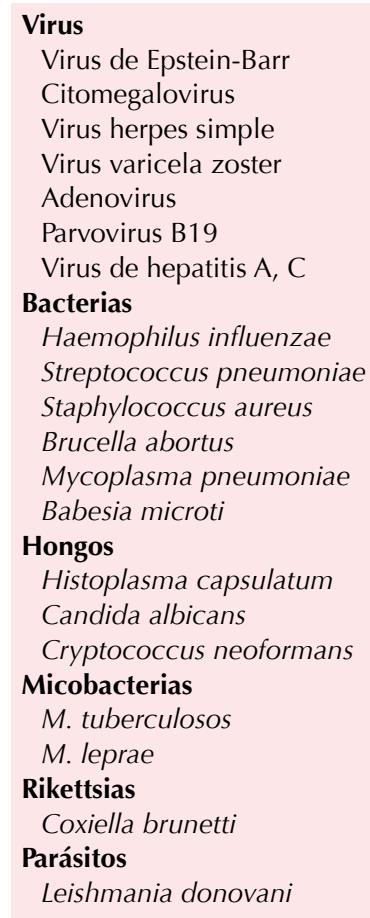

Histiocito propuso los criterios diagnósticos que permanecen como el patrón de referencia para la identificación del síndrome (Cuadro 2). ${ }^{4}$ Evalúan la función citotóxica de las células NK y del receptor alfa soluble para interleucina 2 (IL-2); ${ }^{5}$ sin embargo, en etapas tempranas hay ausencia de hemofagocitos y para el diagnóstico se incluyeron algunos marcadores de activación de macrófagos y de células dendríticas como la ferritina. ${ }^{6}$

Comunicamos el caso de un lactante con hepatitis y síndrome hemofagocítico asociado.

\section{CASO CLÍNICO}

Paciente masculino de un año 11 meses de edad, previamente sano, cuyos familiares negaron antecedentes patológicos. El paciente fue Ilevado a nuestro hospital por un padecimiento de siete días de evolución con rinorrea hialina, congestión nasal, fiebre no cuantificada, dolor abdominal, ictericia, vómito, coluria, petequias y melena. Ingresó somnoliento, ictérico, con equimosis difusas, hepatomegalia y esplenomegalia. Leucocitos 900, hemoglobina $8.7 \mathrm{~g} /$ $\mathrm{dL}$, neutrófilos totales 200, plaquetas 1,000 ; alanino aminotransferasa $415 \mathrm{U} / \mathrm{L}$, aspartato

Cuadro 2. Criterios diagnósticos de linfohistiocitosis hemofagocítica

1. Enfermedad familiar-defecto genético conocido

2. Criterios clínicos y de laboratorio (cinco de ocho criterios)

Fiebre

Esplenomegalia

Citopenia en dos o más líneas celulares

- Hemoglobina $<9 \mathrm{mg} / \mathrm{dL}$

- Neutrófilos <1,000 células

Hipertrigliceridemia, hipofibrinogenemia o ambas

- Triglicéridos

- Fibrinógeno

Ferritina $\geq 500 \mathrm{mcg} / \mathrm{L}$

CD 25 soluble $\geq 2,400 \mathrm{U} / \mathrm{mL}$

Actividad disminuida o ausente de células NK

Hemofagocitos en médula ósea, frotis de sangre periférica o linfonodos 
aminotransferasa 2,481 U/L, gammaglutamiltranspeptidasa $463 \mathrm{U} / \mathrm{L}$, deshidrogenasa láctica 19,428 U/L, fosfatasa alcalina $512 \mathrm{U} / \mathrm{L}$, bilirrubina total $8.2 \mathrm{mg} / \mathrm{dL}$, bilirrubina directa 7.0 $\mathrm{mg} / \mathrm{dL}$, tiempo de protrombina 14.6 segundos, tiempo parcial de tromboplastina 40.5 segundos; anticuerpos IgM-HAV neg; AgHBs IgM neg; hepatitis $\mathrm{C}$ neg; toxoplasmosis $\mathrm{IgM}(-) 3 \mathrm{mg} /$ $\mathrm{dL}$, IgG (+) $14.5 \mathrm{UI} / \mathrm{mL}$; CMV IgM (-) $8 \mathrm{mg} / \mathrm{dL}$, $\operatorname{lgG}(+) 81.2 \mathrm{UI} / \mathrm{mL}$ rubéola $\operatorname{lgM}(-) 10 \mathrm{mg} / \mathrm{dL}$, IgG (-); VEB VCA IgM (-), VCA IgG (+) 77.4 UI/ mL, EBNA-1 IgG (+) $501 \mathrm{Ul} / \mathrm{mL}$, VEB AgC IgM (-), EAg $\lg \mathrm{G}(-)$; los hemocultivos no mostraron desarrollo a los cinco días. Ante la sospecha de origen autoinmunitario de la enfermedad se aplicaron tres bolos de metilprednisolona; el paciente continuó con melena, fiebre y datos de respuesta inflamatoria sistémica. Ingresó a la unidad de terapia intensiva con diagnóstico de choque séptico de foco pulmonar. Tuvo crisis convulsiva tónico-clónico generalizada con tomografía de cráneo normal. Se administró dosis única de gammaglobulina intravenosa $2 \mathrm{~g} / \mathrm{kg}$, el lactante persistió febril con pancitopenia. Los marcadores para síndrome hemofagocítico reportaron ferritina de 18,902.70 ng/mL, triglicéridos de 380 $\mathrm{mg} / \mathrm{dL}$ y el aspirado de médula ósea no confirmó células hemofagocíticas. Se inició tratamiento con dexametasona a $10 \mathrm{mg} / \mathrm{m}^{2}$ de superficie corporal/ día y ciclosporina $3 \mathrm{mg} / \mathrm{kg} / \mathrm{día}$. Persistió febril con datos de dificultad respiratoria, requirió soporte ventilatorio mecánico, tuvo deterioro hemodinámico, coagulación intravascular diseminada, insuficiencia orgánica múltiple y paro cardiorrespiratorio sin respuesta a maniobras avanzadas de reanimación.

\section{DISCUSIÓN}

Se comunica el caso de un lactante con ictericia, dolor abdominal, vómito y ataque al estado general, cuadro sugerente de un proceso infamatorio hepático, bioquímicamente con características de afectación hepatocelular intensa con elevación de deshidrogenasa láctica que sugería un carácter inflamatorio parenquimatoso, la elevación de la gammaglutamil transpeptidasa y fosfatasa alcalina reflejaban discreta afectación canalicular; el paciente tuvo incremento en la bilirrubina directa que introducía un patrón colestásico; con estas características clínicas y bioquímicas se consideró que cumplía con criterios diagnósticos de hepatitis de origen a determinar con patrón colestásico, se inició el abordaje y se descartaron las causas más frecuentes en nuestro país, como la hepatitis A; se solicitaron perfiles infecciosos de acuerdo con la edad del paciente; sin embargo, por la evolución tórpida y la trombocitopenia asociada que condicionaba sangrado a distintos niveles, al descartar el origen infeccioso se consideró el origen inmunológico de la enfermedad y se decidió tratar como hepatitis autoinmunitaria; debido a la mala evolución consideramos el diagnóstico de síndrome hemofagocítico e iniciamos tratamiento inmunosupresor.

El diagnóstico de síndrome hemofagocítico requiere la existencia de citopenias y hemofagocitosis en la médula ósea, pero su ausencia no descarta el diagnóstico. ${ }^{7}$ La trombocitopenia seguida de leucopenia es el hallazgo inicial más frecuente de síndrome hemofagocítico asociado con hepatitis; ${ }^{8}$ algunas explicaciones propuestas son la existencia de inmunocomplejos circulantes y la elevación persistente de citocinas proinflamatorias durante la enfermedad, como IL-1b, factor de necrosis tumoral alfa, IL-6, IL-8, receptor de IL-2, que promueven la activación de macrófagos. ${ }^{9}$

Los pacientes con síndrome hemofagocítico cursan con esplenomegalia, incluso ésta se considera un criterio diagnóstico; en el curso de la enfermedad hay alteraciones en las pruebas de función hepática debido al daño celular secundario a la activación de macrófagos y hemofagocitosis por el sistema retículo-endotelial. 
En el caso comunicado, el paciente manifestó inicialmente su padecimiento con hepatitis aislada, referida como inflamación hepática, traducida como elevación de las enzimas hepáticas, esta inflamación es común en la aparición inicial; sin embargo, la manifestación únicamente hepática es poco frecuente. ${ }^{10}$

En 2004, la Sociedad del Histiocito modificó el tratamiento al incluir la administración de ciclosporina desde el inicio, incluso si las infecciones o citopenias no se han curado. ${ }^{11}$

El síndrome hemofagocítico debe sospecharse en pacientes que cursan con antecedente familiar o infecciones de cualquier causa que muestren mala evolución, con persistencia de fiebre, citopenias, datos de respuesta inflamatoria sistémica, insuficiencia orgánica múltiple sin un foco infeccioso evidente, porque el diagnóstico oportuno contribuye al tratamiento adecuado y mejor pronóstico.

\section{REFERENCIAS}

1. Mashuku S. Differential diagnosis of hemophagocytic syndrome: underlying disorders and selection of the most effective treatment. Int J Hematol 1997;66:135-151.
2. Janka GE, Schneider EM. Modern management of children with haemophagocytic lymphohistiocytosis. Br J Haematol 2004;124:4-14.

3. Kumakura $\mathrm{S}$, Ishikura $\mathrm{H}$, Kondo $\mathrm{M}$, et al. Autoimmuneassociated hemophagocytic syndrome. Mod Rheumatol 2004;14:205-215.

4. Henter JI, Horne A, Arico M, Egeler RM, et al. HLH-2004: Diagnostic and therapeutic guidelines for hemophagocytic lymphohistiocytosis. Pediatr Blood Cancer 2007;48:124131

5. Lin TF, Ferlic-Stark LL, Allen CE, Kozinetz CA, McClain KL. Rate of decline of ferritin in patients with hemophagocytic lymphohistiocytosis as a prognostic variable for mortality. Pediatric Blood \& Cancer 2011;56:154-155.

6. Canna SW, Behrens EM. Not all hemophagocytes are created equally: appreciating the heterogeneity of the hemophagocytic syndromes. Curr Opin Rheumatol 2012;24:113-118.

7. Filipovich $\mathrm{AH}$. Hemophagocytic lymphohistiocytosis: a lethal disorder of immune regulation. J Pediatr 1997;130:337-338.

8. Gundersen SG, Bjoerneklett A, Bruu JN. Severe erythroblastopenia and hemolytic anemia during a hepatitis $A$ infection. Scand J Infect Dis 1989;21:225-228.

9. Murohashi I, Yoshida K, Ihara N, et al. Serum levels of Thl/ Th2 cytokines, angiogenic growth factors, and other prognostic factors in young adult patients with hemophagocytic syndrome. Lab Hemat 2006;12:71-74.

10. Amin N, Shah I, Bhatnagar S. Hemophagocytic lymphohistiocytosis $(\mathrm{HLH})$ in children presenting as liver disease. $J$ Clin Exp Hepatol 2014;4:175-177.

11. Jordan M, Allen C, Weitzman S, et al. How I treat hemophagocytic lymphohistiocytosis. Blood 2011;118:4041-4052. 